\title{
CERN n_TOF Facility and its Possible Impact on Producing Nuclei with Large Neutron Excess
}

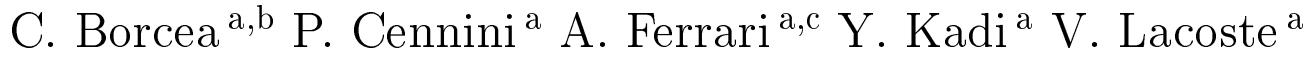 \\ E. Radermacher ${ }^{\mathrm{a}} \mathrm{V}$. Vlachoudis ${ }^{\mathrm{a}}$ \\ ${ }^{a}$ CERN, SL Division, EET Group, CH-1211 Geneve 23, Switzerland \\ ${ }^{\mathrm{b}}$ IFIN-HH, PO Box MG-6, Bucharest-Magurele, Romania \\ ${ }^{\mathrm{c}}$ INFN Milan, Italy
}

\begin{abstract}
The n_TOF facility at CERN is a source of neutrons produced by spallation of $20 \mathrm{GeV} / \mathrm{c}$ protons onto a lead target. The outstanding characteristics of this source (very high intensity, $200 \mathrm{~m}$ flight path, wide spectral function) make it an extremely useful tool for studying neutron induced reactions. The neutrons in the energy region from 1 to $10 \mathrm{MeV}$ are particularly interesting for producing nuclei with large neutron excess by fission. The onset of an asymmetric fission mode at these energies seems to considerably enhance the yield of neutron rich species. Therefore, a method could emerge for producing neutron rich rare earth nuclei in a region up to now inaccessible.
\end{abstract}

The n_TOF is a CERN spallation neutron facility at CERN. Its description, including the main characteristics and also the physics program to be accomplished can be found [1] in the TOF Proposal and the recent Technical Design Report. Here, only some relevant parameters for the following discussion will be given. All the parameters of the installation resulted from a detailed Monte Carlo simulation that made use of the advanced FLUKA-EA-MC[2] programs.

This paper is organized as follows: firstly some parameters of n_TOF having a particular relevance for the coming discussion will be presented. Then, some recent data concerning the mass distributions of fragments from fission of ${ }^{238} \mathrm{U}$ and ${ }^{237} \mathrm{~Np}$ with neutrons of $5 \mathrm{MeV}$ energy will be reviewed with emphasis on the production of exotic species. Corresponding productivities will be evaluated for the TOF conditions. They will be also compared to the recent results obtained at GSI by fissioning an ${ }^{238} \mathrm{U}(750 \mathrm{~A} \cdot \mathrm{MeV})$ incident beam on $\mathrm{Pb}$ and $\mathrm{Be}$ targets. Conclusions will be drawn concerning the potential of TOF to produce/study exotic nuclei.

The $20 \mathrm{GeV} / \mathrm{c}$ beam from the CERN-PS will impinge on a spallation target made of high purity lead (a solid block with the dimensions of $60 \times 80 \times 80 \mathrm{~cm}^{3}$ ). 
The target is immersed in a water pool that serves also as a moderator and which flattens the spectral shape of the produced neutrons. A flight path of about $200 \mathrm{~m}$ follows after the target and the moderator water layer $(5 \mathrm{~cm}$ thick), allowing a precise energy determination of the neutrons by time of flight. The direction of this flight path makes an angle of $10^{\circ}$ with respect to the incoming proton beam direction. A window with a diameter of $80 \mathrm{~cm}$ separates the target-moderator assembly from the flight path tube from which the air is evacuated. The spatial distribution of neutrons at the exit of the window as expected from the simulations is shown in fig.1a [3]. If only the neutrons with energies in the interval $1-10 \mathrm{MeV}$ are selected, then the distribution is narrower and preserves a memory of the initial proton beam direction (see fig.1b). The simulations indicate that for each incident proton, about 70 neutrons are produced that come out of the window. Of these, about one neutron having the energy in the indicated domain will be placed in a disk with a diameter of $10 \mathrm{~cm}$ centered on the peak in fig.1b. As the PS can deliver $710^{12}$ protons in a pulse (with a time width $\sim 7 n s$ ), some $610^{12}$ such neutrons will be created per proton pulse.

In the following one will briefly review some recent experimental results concerning the fission of ${ }^{238} \mathrm{U}$ with neutrons of energies between 1 and 5.5 $\mathrm{MeV}$ [4]. The authors of [4] measured the mass and total kinetic energy distributions for fragments resulting from ${ }^{238} \mathrm{U}$ fission with neutrons of various energies and made a multimodal analysis. Their results show that the contribution of the so called standard 2 mode is responsible for the production of masses placed on the wings of the well known two humped distribution. Moreover, the contribution of this mode increases with the neutron incident energy and so does its width. This tendency has been followed up to $5.8 \mathrm{MeV}$; it is likely that it will be preserved for some additional 1-2 MeV. For higher energies, multiple chance fission sets in and complicates the analysis. From the files kindly provided by the author one can see mass splits as asymmetric as 63 and 176 at yield levels of about $10^{-3 \%}$. The charge yield curves measured by [5] extend from $Z=30$ to $Z=62$ but the yield values at the extremes are difficult to evaluate on the linear scale. The distributions presented in [4] are the initial ones, in the sense that they are corrected for the evaporated neutrons. This correction can amount to two neutrons for the heavy fragment and 0.5 neutrons for the light fragment. One should remark at this point that such asymmetric splits lead imperatively to nuclei far from stability. On the isobaric lines 63 and 174 all $Z$ combinations adding up to 92 give rise to very exotic nuclei. Perhaps the less exotic of all is the pair ${ }^{63} \mathrm{Cr}-{ }^{174} \mathrm{Er}$. Trying to come close to stability with one fragment will automatically make its partner more exotic. The above mentioned yield will be distributed among different pairs of fragments corresponding to different $Z$ splits and the more exotic will be one of the partners, the lower will be the yield. As charge measurements are not easy to perform at this sensitivity level one can only suppose that the products with very asymmetric mass splits will be distributed over a limited number of charge splits and that moving away from stability with one partner 
will imply a sharp decrease in the yield, e.g. one order of magnitude per step. In terms of cross sections, taking for ${ }^{238} \mathrm{U}$ a fission cross section of $0.66 \mathrm{~b}$, the mass split $63-174$ will appear with a cross section of $0.6610^{-5}=6.6 \mu \mathrm{b}$. Considering another 3 orders of magnitude decrease for more exotic $Z$ splits one still have a cross section of the order of $1 \mathrm{nb}$. This value is comparable to the cross sections for production of exotic species recently measured at GSI [6] in the fission of ${ }^{238} \mathrm{U}$ projectiles of $750 \mathrm{~A} \cdot \mathrm{MeV}$ on a Be target. In particular, the mass split that lead to the observation of ${ }^{78} \mathrm{Ni}$ in that experiment, ${ }^{78} \mathrm{Ni}-{ }^{159} \mathrm{Gd}$ is not very exotic; instead, the charge split is a very exotic one and it has paid the price of a very low cross section.

${ }^{237} \mathrm{~Np}$ is also a good candidate for producing exotic nuclei by fission. Siegler et al. [7] reported data that present a broad mass distribution and a similar multimodal analysis. The advantage of ${ }^{237} \mathrm{~Np}$ is a higher fission cross section $(\sim 1 b)$ but the disadvantages are its lower $N / Z$ ratio and its radioactivity and cost.

Some estimations can be made for the production of exotic species using the induced fission of ${ }^{238} \mathrm{U}$ by the spallation neutrons of the TOF installation. For a disk-shaped $U C_{2}$ target with a diameter of $10 \mathrm{~cm}$ and a thickness of $10 \mathrm{~mm}$ placed on the position of maximum flux for neutrons of 1-10 MeV described above, about $610^{12}$ neutrons will traverse the target at each PS pulse. Taking a mean fission cross section value of $0.66 \mathrm{~b}$, one expects about $10^{11}$ fission events per burst. If the yield of very asymmetric mass splits is taken (following [4]) as $10^{-5}$, then $10^{6}$ such cases will occur at each burst. Taking out another 3 orders of magnitude for also producing exotic $Z$ splits one arrives at $10^{3}$ per burst. The experience accumulated at ISOLDE [8] shows that the extraction efficiency for rare earths vary between 10 and $30 \%$ while the post-acceleration has a $10 \%$ efficiency. This means that in an ISOLDE type installation, about 10 very exotic species per burst can be expected (whose production cross section is about $1 \mathrm{nb}$ ). The lifetimes in the region of interest are expected to be rather long and therefore no additional decay losses are considered. In this estimative calculation, the thermal fission yield has not been considered, though its contribution is very important in view of the high flux for thermal neutrons. For less exotic mass and charge splits this contribution will add to the above described one, such that, for "moderately" exotic nuclei (yields $\sim 10^{-3}$ ), the productivity may reach as many as $310^{6}$ per pulse. The energy release of the produced fission events in the target (including thermal fission) will amount to about $7 \mathrm{~J} /$ pulse. In one day's irradiation, as many as 5000 pulses can be used which casts rather optimistic perspectives for attaining even such exotic splits as ${ }^{60} \mathrm{Ca}-{ }^{177} \mathrm{Hf}$. A highly enhanced neutron flux (about one order of magnitude) can be obtained by inserting the $U C_{2}$ target assembly into the lead spallation block. This solution is currently under study.

In conclusion, some perspectives have been presented for using the new TOF facility at CERN for producing nuclei far from stability. In particular, this method could provide a bridge to fill up the "gulf" existing in the chart of nuclides on the neutron rich side of the rare earth region. At the other 
extreme, the attempt to reach ${ }^{60} \mathrm{Ca}$, if successful, could cast a new light on the persistence of shall closure at very high neutron excess.

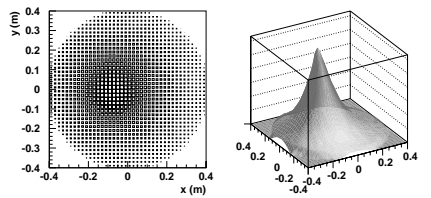

(a)

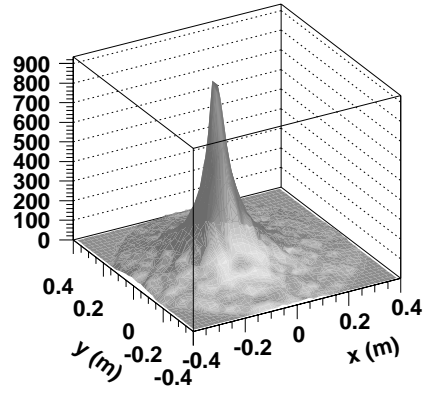

(b)

Fig. 1. Neutron spatial distribution at the exit of the window: a) all the neutrons; b) neutrons with energies in the range from 1 to $10 \mathrm{MeV}$.

\section{References}

[1] TOF Proposal, CERN/ISPSC/99-8 SPC/P 310, TOF Technical Design Report, CERN/INTC/2000-04.

[2] A. FAssó et al. in "Intermediate Energy Nuclear Data: Models and Codes", Proceedings of a Specialists Meeting, Issy les Moulineaux (France) 30 May 1-June 1994, p.271, published by OECD, 1994 and references therein C. RubBia et al. Conceptual Design of a Fast Neutron Operated High Power Energy Amplifier CERN/AT/95-44(ET)

[3] V. Vlachoudis et al., CERN/ET/Internal Note/2000-06

[4] F. Vives et al., Nucl. Phys. A 662 (2000) 63

F. Vives, These, Univ. Bordeaux, 1998

[5] G. Rudstam, Proc. Int. Conf. On Nuclear Data for Science and Technology, Gatlinburg, 1994 (ed J.K. Dickens)

[6] M. Bernas et al., Phys. Lett. B 450 (1997) 111

[7] P. Siegler et al., Nucl. Phys. A 594 (1995) 45

[8] H. RAVN, private communication 